\title{
LHomme
}

L'HOMME Revue française d'anthropologie

$213 \mid 2015$

Varia

\section{Nicole-Claude Mathieu (1937-2014)}

\section{Marie-Élisabeth Handman}

\section{(2) OpenEdition}

Journals

Édition électronique

URL : http://journals.openedition.org/lhomme/23654

DOI : 10.4000//homme.23654

ISSN : 1953-8103

\section{Éditeur}

Éditions de l'EHESS

\section{Édition imprimée}

Date de publication : 26 février 2015

Pagination : 19-32

ISSN : 0439-4216

\section{Référence électronique}

Marie-Élisabeth Handman, « Nicole-Claude Mathieu (1937-2014) ». L'Homme [En ligne], 213 | 2015, mis en ligne le 25 février 2017, consulté le 10 décembre 2020. URL : http://journals.openedition.org/ Ihomme/23654 ; DOI : https://doi.org/10.4000//homme.23654 


\section{Nicole-Claude Mathieu (1937-20|4)}

\section{Marie-Élisabeth Handman}

Elle s'appelait Nicole-Claude mais, au Laboratoire d'anthropologie sociale auquel elle appartenait depuis 1971, nous l'appelions Nicole. Son absence se fait cruellement sentir pour la plupart d'entre nous, femmes surtout, qui avons tant appris à son contact et partagé tant de moments d'amitié. Je repense particulièrement à ces fins d'après-midi quand, lorsque cela était encore permis, nous nous retrouvions à deux ou trois pour fumer dans son bureau (dans lequel on se frayait un chemin à travers un rideau de fumée) ou dans le mien (qui n'était pas moins opaque) et poursuivions dans la convivialité notre journée de travail jusque tard dans la soirée. Car Nicole était une travailleuse infatigable et d'une exigence féroce vis-à-vis d'elle-même.

Ce sens du travail et du travail bien fait, c'est sa grand-mère paternelle, ouvrière depuis l'âge de treize ans, qui le lui avait transmis. Nicole, née le 28 novembre 1937 en Vendée, fut en effet élevée par cette femme à laquelle on l'avait confiée, à la fois pour la mettre à l'abri durant la guerre, mais aussi parce qu'il fallut l'éloigner de sa mère tuberculeuse, par peur de la contagion. Elle vécut ainsi une partie de son enfance dans le milieu de la petite paysannerie prolétarisée de l'Est de la France, dont elle gardera une solide conscience de classe sociale, puis proche du milieu ouvrier de l'usine que dirigeait son père, ingénieur, qui, disait-elle, lui avait donné le goût des découvertes scientifiques. 
Nicole ne parlait que fort peu de sa vie personnelle, il est vrai tôt émaillée de deuils, probablement trop douloureux à évoquer. Toutefois, dans la brève page qui ouvre L'Anatomie politique 2 (2014), page qu'elle a écrite peu de temps avant sa mort survenue le 9 mars 2014, et qu'elle a intitulée "Ma vie» mais rédigée à la troisième personne du singulier comme pour mettre cette vie à distance, elle relate que, "opportunément dépourvue de frère, éduquée dans un collège laïque de filles par des professeurs femmes, entourée d'amitiés féminines sans complications hétérosexuelles, elle grandit donc en être humain " (Ibid.: 5, mes italiques). Cette dernière proposition, à première vue énigmatique, prend tout son sens, nous le verrons, à la lumière de l'ensemble de son ouvre.

Son immense curiosité intellectuelle l'a conduite à mêler études de lettres, de sociologie et d'ethnologie, entre 1956 et 1962. En 1960, elle fut stagiaire auprès du Haut-Commissariat au Plan de la République centrafricaine à Bangui, ce qui constituera sa seule expérience en terrain " exotique ». De 1960 à 1965, elle collabora à des recherches de sociologie urbaine, sociologie des femmes et sociologie du racisme au sein du Groupe d'ethnologie sociale dirigé par Paul-Henry Chombart de Lauwe. Elle devint ensuite, entre 1966 et 1969, rédactrice de la revue de l'Unicef, Les Carnets de l'enfance/Assignment Children, avant de passer un an au Centre d'études sociologiques de Paris en qualité d'assistante de recherche contractuelle, chargée du devenir professionnel des étudiants. C'est en 1971 que Nicole entra au Laboratoire d'anthropologie sociale, alors dirigé par Claude Lévi-Strauss, avec le grade de chef de travaux (enseignantschercheurs subalternes) et la fonction de secrétaire de rédaction de la revue L'Homme auprès de Jean Pouillon, puis de la collection des "Cahiers de L'Homme ", tâches qu'elle assumera jusqu'en 1983 pour la première et 1990 pour la seconde.

Ses fonctions éditoriales à la revue L'Homme ne l'empêchèrent pas de rédiger et de publier elle-même au moins un article par an, puis plusieurs à partir de 1977, tout en participant, avec Christine Delphy et d'autres ${ }^{1}$, à la création de la revue Questions féministes. À cela s'ajoutèrent des traductions de l'anglais, la révision des traductions en anglais ou en allemand de ses propres textes - textes qui, pour nombre d'entre eux, ont été traduits dans pas moins de sept langues -, et la direction de Diplômes de l'EHEss. Ce diplôme propre à l'École sanctionne, rappelons-le, la rédaction d'un mémoire permettant à des personnes ne possédant pas forcément le bac,

1. En 1980, pour des raisons tenant moins à des divergences théoriques qu'à des incompatibilités d'humeur, Nicole-Claude et quelques autres, dont Colette Capitan et Monique Wittig, quittèrent Questions féministes. Un an plus tard, Christine Delphy lançait Nouvelles Questions féministes avec Claude Hennequin et Emmanuèle de Lesseps. 
mais ayant une expérience professionnelle ou personnelle singulière, de s'initier en trois ans à la recherche scientifique voire, au-delà, de s'inscrire en thèse. Ce modèle de formation, atypique dans le paysage de l'enseignement supérieur français, ne pouvait manquer de séduire quelqu'un d'aussi altruiste que Nicole, qui s'investit pleinement dans son rôle d'encadrante, faisant preuve, avec ses élèves, d'une patience infinie ou d'une certaine sévérité lorsque cela s'avérait nécessaire. Car cette femme, qui était d'une générosité sans borne, savait aussi ne pas mâcher ses mots!

Dans les années 1970 et jusqu'en 1998², travailler sur les rapports sociaux de sexe et être féministe de surcroît étaient extrêmement mal vu à l'EHESS : les chercheuses répondant à ces critères étaient moins jugées sur leur valeur scientifique que sur leur engagement militant. Or, NicoleClaude s'inscrivait indéniablement dans cette catégorie en tant qu'anthropologue engagée dans le combat pour les droits des femmes, s'efforçant, en dehors de toute organisation politique ou syndicale, de lutter contre les injustices sociales à l'intérieur comme à l'extérieur de son institution ${ }^{3}$. Cela explique sans doute pourquoi elle stagna au $\sigma^{\mathrm{e}}$ échelon du corps des chefs de travaux jusqu'en 1990, alors même que l'École des hautes études en sciences sociales s'était lancée, dès les années 1980, dans une politique de transformation de cette catégorie intermédiaire au point de la faire disparaitre après 1985. Longtemps demeurée au bas de l'échelle des enseignants-chercheurs, Nicole-Claude fit partie des tout derniers à se voir promus maîtres de conférences, ce qui fait donc suspecter une différence de traitement en sa défaveur. C'est ainsi que, lassée des sarcasmes de ses collègues masculins, considérant qu'elle avait mieux à faire que de passer l'habilitation à diriger des recherches - qui, pourtant, lui aurait permis d'encadrer des doctorants - et persuadée que tenter de se faire élire directrice d'études serait voué à l'échec, elle renonça à toute forme de progression de carrière et resta maîtresse de conférences jusqu'à sa retraite. Pourtant, et grâce à sa renommée internationale, la reconnaissance vint de ses pairs étrangers qui n'avaient, quant à eux, pas attendu sa promotion dans la hiérarchie académique française pour l'honorer: elle reçut, le 15 juin 1996, le titre de Docteure en sciences sociales honoris causa par l'Université Laval au Québec, titre qui n’avait été décerné qu’à un seul Français avant elle : Claude Lévi-Strauss !...

2. L'année 1998, celle de l'adoption du Pacte civil de solidarité (PACS) par l'Assemblée nationale, a marqué un tournant radical pour les enseignants de l'École : il n'était plus ridicule de s'intéresser aux questions de l'orientation sexuelle, ni même à ce que Nicole-Claude Mathieu a elle-même défini comme les "rapports sociaux de sexe".

3. Par l'intermédiaire de ses écrits bien sûr, mais aussi par l'intermédiaire d'actes concrets, notamment lorsqu'elle hébergea des femmes battues avant que des refuges ne soient mis à leur disposition, ou lorsqu'elle milita au sein d'une association de défense des prostituées. 
Nicole-Claude Mathieu fut une pionnière du féminisme matérialiste. Il s'agit d'un courant féministe d'inspiration marxienne qui s'élève contre la doxa communiste des années 1970 en France, selon laquelle la disparition du capitalisme et de la domination de classe entraînerait automatiquement la disparition de la domination de sexe. Or, pour les féministes matérialistes, le patriarcat n'est en rien un produit du seul capitalisme, même si ce dernier peut en accentuer les effets délétères : la domination patriarcale des femmes traverse toutes les classes et existe aussi dans les sociétés sans classe. Il convient donc de combattre simultanément patriarcat et capitalisme. Par ailleurs, ce féminisme affirme qu'il existe, non pas une différence ontologique, mais une simple différenciation des sexes du fait de l'idéologie et des pratiques. En quoi il s'oppose au féminisme différencialiste ou essentialiste. Tout le travail de Nicole-Claude Mathieu a consisté à déconstruire les idéologies qui sous-tendent ou justifient l'"oppression des femmes" - expression qu'elle préférait à celle de "domination masculine ", qui, estimait-elle, fait la part trop belle aux dominants. Ainsi, à la fin de "Ma vie », elle écrivait :

"L'ethnologie est sa discipline d'élection, car seule apte à révéler la diversité mais aussi l'unité (et la perversité) de l'esprit humain, et son terrain de recherche l'observation hallucinée de l'amplitude de l'oppression des femmes» (Mathieu 2014: 6).

Forte de sa double formation d'anthropologue et de sociologue, elle a pu analyser ce qu'il y a de commun aux sociétés les plus diverses, occidentales ou non, dans les manières d'opprimer les femmes ou de (dé)nier cette oppression. Pour elle comme pour les autres féministes matérialistes (Colette Guillaumin, Colette Capitan, Paola Tabet, Christine Delphy...), les sexes sont socialement divisés en deux classes dont l'une, celle des hommes, exploite l'autre, celle des femmes.

La quasi-totalité des écrits de Nicole-Claude Mathieu a été rééditée dans deux recueils, intitulés respectivement L'Anatomie politique. Catégorisations et idéologies du sexe $(1991)^{4}$ et L'Anatomie politique 2. Usage, déréliction et résilience des femmes (2014).

Le premier de ces recueils regroupe des textes prononcés ou écrits entre 1971 et 1989 , dont quatre articles de critique épistémologique de la sociologie et de l'ethnologie : notamment, «Critiques épistémologiques de la problématique des sexes dans le discours ethno-anthropologique" (Mathieu 1991: 75-130) ${ }^{5}$. Nicole-Claude Mathieu s'y efforçait de

4. Une seconde édition, augmentée d'un tableau synoptique présentant les trois modes de l'identité sexuelle/sexuée/de sexe, est parue en 2013.

5. Il s'agit d'un rapport préparé par Nicole-Claude Mathieu pour l'Unesco et présenté à la Réunion internationale d'experts: "Réflexions sur la problématique féminine dans la recherche et l'enseignement supérieur" (Lisbonne, 17-20 septembre 1985). 
démontrer les biais androcentriques de la pensée dominante, qui invisibilisent la femme en ne la définissant que par rapport au modèle général qu'est l'homme ou qui, lorsqu'ils lui font une place, lui nient tout statut de sujet social ou culturel pour ne leur reconnaître que celui de sujet sexué voire, diraient les linguistes, d' "animé non humain» ou de "non animé " ${ }^{6}$. Ces expressions de l'animéité sont inspirées d'un article de Claire Michard-Marchal et Claudine Ribéry (1985), qui proposaient une analyse sociolinguistique du discours occidental sur les sexes et que Nicole-Claude publia la même année dans l'ouvrage collectif L'Arraisonnement des femmes. Essais en anthropologie des sexes (Mathieu, ed. 1985). Cet ouvrage est probablement celui qui, avec quelques entrées de dictionnaires ${ }^{7}$, lui valut le plus de sarcasmes de la part de nos collègues masculins. La seule mention du titre les faisait hurler de rire. Est-ce la raison pour laquelle ce livre, vite épuisé, n’a jamais été réédité ? Fort heureusement, sa contribution "Quand céder n'est pas consentir: des déterminants matériels et psychiques de la conscience dominée des femmes, et de quelques-unes de leurs interprétations en ethnologie » (Ibid. : 169-245) fut republiée dans L'Anatomie politique (Mathieu 1991: 131-226). Il fait partie de ces textes qui, avec "Identité sexuelle/sexuée/de sexe? Trois modes de conceptualisation du rapport entre sexe et genre" (Mathieu 1989) ${ }^{8}$, ont connu un formidable retentissement international et sont devenus incontournables dans l'enseignement de la sociologie et de l'anthropologie des sexes - puis de l'anthropologie sociale tout court. À ces textes fondamentaux, il convient d'ajouter deux articles de Paola Tabet souvent cités par Nicole-Claude, «Fertilité naturelle, reproduction forcée » paru dans L'Arraisonnement (Mathieu, ed. 1985: 61-146) et "Les mains, les outils, les armes " paru dans L'Homme (Tabet 1979), qu' elle contribuera même, avec sa générosité et sa méticulosité habituelles, à faire republier en 1998 (Tabet 1998).

Revenons sur les "Critiques épistémologiques de la problématique des sexes dans le discours ethno-anthropologique" (Mathieu 1991 : 75130). Ce rapport rédigé par Nicole-Claude Mathieu était une analyse des écrits anthropologiques de féministes qui, déterminées à rendre visibles les femmes et leurs travaux tout en démontrant leur autonomie par une dénonciation de l'androcentrisme des spécialistes des sciences sociales, en viennent à considérer qu'il y a une "égalité dans la différence " et,

6. Le fait qu'elle fut peu entourée d'hommes explique pourquoi Nicole-Claude a eu le sentiment d'avoir grandi «en être humain» (cf. cette phrase énigmatique que je citais au début de l'article).

7. Notamment les entrées «Féministes (études) et anthropologie " et "Sexes (différenciation des) ", in Pierre Bonte \& Michel Izard, eds (1991: 275-278 et 660-664).

8. Republié dans L'Anatomie politique (Mathieu 1991 : 227-268). 
par conséquent, à occulter les rapports de pouvoir, le fait que le pouvoir des hommes repose largement sur l'exploitation des femmes et qu'il convient de faire non pas une anthropologie des femmes mais une anthropologie des sexes et de leurs rapports sociaux. L'anthropologie des femmes reste tributaire de l'essentialisme qui les veut par nature différentes des hommes. Or, on l'a vu, pour Nicole-Claude Mathieu - d'ailleurs d'accord en cela avec les biologistes -, il n'y a pas différences (biologique, ontologique) mais différenciations (sociale, idéologique) des sexes. Dans tous ses articles, elle martelait que la répartition sexuée des tâches dans les diverses sociétés n'a rien à voir avec l'impossibilité pour les femmes de se déplacer loin du foyer (elle donnait de nombreux exemples allant à l'encontre de cette croyance), mais avec la volonté des hommes de les contrôler à des fins reproductrices, au double sens de reproductrices de l'espèce et de la force de travail. Elle dénonçait donc l'idée d'une complémentarité dans la division sexuelle du travail que, pour cette raison, elle préférait appeler division socio-sexuée du travail. À l'époque où ces articles furent écrits, les féministes différencialistes étaient majoritaires et la position de NicoleClaude Mathieu, qui admettait les apports factuels de leurs travaux, apparut en France comme trop radicale pour être prise au sérieux. Pourtant, sa pensée puisait aussi dans l'œuvre de Claude Lévi-Strauss, lui reconnaissant d'avoir mis en lumière le fait que la prohibition de l'inceste " opère seule une refonte des conditions biologiques de l'accouplement et de la procréation. Elle ne permet aux familles de se perpétuer qu'enserrées dans un réseau artificiel d'interdits et d'obligations " (Lévi-Strauss 1983: 83 , je souligne) ${ }^{9}$. Parmi ces obligations, outre la division sexuelle arbitraire du travail, figure en bonne place l'hétéronormativité, en sorte que, en 1975, Gayle Rubin écrira: "Lévi-Strauss est dangereusement près de dire que l'hétérosexualité est un processus institué " (in Rubin 1998 : 49) ${ }^{10}$. Dans un article de 2010, Nicole-Claude, en collaboration avec Martine Gestin ${ }^{11}$, s'attachera de son côté à démontrer que Lévi-Strauss est antinaturaliste dans sa conception du genre humain (Gestin \& Mathieu 2010) ${ }^{12}$.

9. Il s'agit de la traduction et de l'adaptation de l'article "The Family", paru en 1956 in Harry L. Shapiro (1956: 261-283).

10. Il s'agit de la traduction par Nicole-Claude de "The Traffic in Women : Notes on the "Political Economy" of Sex » (cf. Rubin 1975), traduction par la suite révisée in Gayle Rubin (2010).

11. Dans les dernières années de sa vie, Nicole-Claude, gravement malade et atteinte d'une dégénération de la macula qui lui rendait la lecture et l'écriture extrêmement difficiles, a trouvé en sa compagne Danielle Charest, écrivaine elle-même disparue en 2011, et en Martine Gestin, anthropologue indianiste, l'aide qui lui était nécessaire pour poursuivre son œuvre.

12. Republié dans L'Anatomie politique 2 (Mathieu 2014 : 109-122). 
Dans l'article «Quand céder n'est pas consentir : des déterminants matériels et psychiques de la conscience dominée des femmes, et de quelques-unes de leurs interprétations en ethnologie " (Mathieu, ed. 1985: 169-245), Nicole-Claude Mathieu souhaitait mettre en évidence le fait que même si elles partagent les idées des hommes sur le fonctionnement de leurs sociétés, les femmes n'ont pas accès à toutes les données mythiques, religieuses, politiques, techniques... qui pourtant les concernent. Leurs connaissances sont limitées et leur vécu des idées partagées differe de celui des hommes. Cette conscience restreinte ne permet donc pas un consentement libre et éclairé. Toutefois, les femmes cèdent face aux dominants qui utilisent toutes formes de violences symboliques, psychologiques ${ }^{13}$ et physiques pour maintenir leur supériorité, contrairement à ce qu'affirmait Maurice Godelier. Ce dernier, en effet, après avoir montré dans les moindres détails la réalité de la domination masculine chez les Baruya, concluait que "des deux composantes du pouvoir la force la plus forte n'est pas la violence des dominants mais le consentement des dominés à leur domination" (1982: 232). Tout au long de son article, Nicole-Claude Mathieu insistait sur la sur-fatigue des femmes et sur la sous-évaluation de la charge de travail pesant sur elles, dues à l'accaparement continu de leur corps pour les travaux des champs, le portage des enfants, de l'eau, du bois, les grossesses imposées ou le sous-équipement technique qui caractérise leur classe de sexe (voir Tabet 1979), à quoi s'ajoute souvent la malnutrition. À quoi s'ajoute aussi la peur de ne pas répondre aux normes imposées par les hommes, surtout quand elles sont contradictoires.

Armée d'une remarquable érudition ethnographique, Nicole-Claude traqua ainsi dans le monde entier les exemples de l'oppression des femmes, principalement dans les sociétés patrilinéaires virilocales, mais également dans les sociétés matrilinéaires à résidences uxorilocales. J'y reviendrai, mais je voudrais souligner ici que le livre collectif consacré à cette question qu'elle dirigea en 2007, Une maison sans fille est une maison morte (Mathieu, ed. 2007), est loin d'être la réponse à une interrogation tardive. Car ce qui caractérise, entre autres, tout l'œuvre de cette chercheuse d'exception, ce sont les fortes intuitions qu'elle eut dès le départ et n'a cessé ensuite de creuser, d'étayer, d'étoffer. Par exemple, dès 1971 elle remarquait que "certaines sociétés ont élaboré trois catégories sociales de sexe: les hommes, les femmes et une troisième sorte d'individus ni

13. Nicole-Claude Mathieu cite entre autres exemples la situation ambivalente des femmes, contraintes à la fois d'obéir aux hommes (en acceptant leurs avances sexuelles) et de leur désobéir (sous peine d'être traitées de "putains"). 
homme ni femme ou à la fois homme et femme, et celle-ci non seulement dans les mythologies mais dans la réalité sociale» $(1971: 27)^{14}$. Ce n'est pourtant qu'en 1982, puis en 1989, qu'elle mit au point l'«Identité sexuelle/sexuée/de sexe : trois modes de conceptualisation du rapport entre sexe et genre " ${ }^{15}$. Cet article est particulièrement complexe parce qu'il tient compte des niveaux à la fois individuel et collectif de ces conceptualisations, des normes et des représentations transgressives ou non, subversives ou non. Rappelons que les trois modes sont définis de la manière suivante :

"Mode I : Identité "sexuelle", basée sur une conscience individualiste du sexe. Correspondance homologique entre sexe et genre : le genre traduit le sexe.

Mode II : Identité "sexuée", basée sur une conscience de groupe. Correspondance analogique entre sexe et genre: le genre symbolise le sexe (et inversement).

Mode III : Identité "de sexe", basée sur une conscience de classe. Correspondance sociologique entre sexe et genre : le genre construit le sexe» (Mathieu 1991 : 231).

Grossièrement résumé, le mode I, dans lequel le référent est une bipartition naturelle et sociale du sexe, correspond au modèle hétérosexuel de nos sociétés occidentales; dans cette optique "naturaliste », l'homosexualité y est une anomalie (notons que Nicole-Claude écrit vingt-cinq ans avant la loi autorisant, en France, le mariage homosexuel). Le mode II, où «le genre est ressenti comme une sorte de mode de vie collectif » (Ibid. : 239), correspondrait aux sociétés qui, traditionnellement, autorisent le mariage entre personnes de même sexe (garçons-épouses azande, mariages entre femmes en Afrique, berdaches nord-américains). Dans le mode III, la bipartition du genre, "conçue comme étrangère à la "réalité" biologique du sexe " et permettant de dire que le genre construit le sexe (Ibid. : 255256), renvoie à des positions idéologiques et politiques, à une identité de résistance au genre en tant qu' « opérateur du pouvoir d'un sexe sur l'autre" (Ibid. : 258).

Nicole-Claude Mathieu classe, un peu rapidement me semble-t-il, les Inuit et les hijras de l'Inde dans le mode I. En effet, certains enfants inuit sont éduqués dans le genre opposé à leur sexe anatomique lorsque le sexe de la personne dont ils reçoivent le nom à leur naissance est différent du leur. Il est vrai qu'à l'âge de la puberté, c'est-à-dire en prévision du mariage et de la procréation, ces enfants "travestis " retrouvent le genre qui correspond à leur sexe, ce qui renvoie bien à la logique "hétérosexuelle » du mode I (Ibid. : 238). Or, précise Bernard Saladin d'Anglure

14. Citation extraite d'un article republié dans L'Anatomie politique (Mathieu 1991 : 17-41).

15. Ces trois modes sont repris dans les deux éditions de l'Anatomie politique (1991, 2013). Cf., supra, note 4. 
(et je suis d'accord avec lui) mais pas Nicole-Claude, les enfants qui ont été élevés dans le genre opposé à leur sexe gardent, une fois adultes, la possibilité d'appartenir aux deux genres, constituant ainsi un troisième sexe social. Quant aux hijras, tout au moins dans la tradition indienne, ils se considèrent comme n'étant ni homme ni femme, sont castrés et se travestissent en femmes afin de servir la déesse à laquelle ils se vouent, ce qui relève, à mon sens, du mode II même s’il est vrai qu'après la colonisation britannique et à l'époque où écrit l'ethnologue sur laquelle s'appuie Nicole-Claude (1989), ils sont devenus des homosexuels prostitués et parfois des transsexuels caractéristiques de l'optique "naturaliste" du mode I. Mais, si elle ne la commente pas, Nicole-Claude ne conteste pas pour autant l'existence chez les Inuit de nombreux intersexes, car, ce qui lui importe avant tout, c'est de démontrer que, quelles que soient les conceptions des rapports entre sexes et genre, les hommes restent dominants et les femmes dominées. Pour n'en donner qu'un seul exemple, elle rappelle que les guerriers azande du Sud-Soudan avaient le droit, en attendant d'être assez riches pour se marier avec une femme, d'épouser de jeunes garçons, alors qu'à l'inverse l'homosexualité des femmes était sévèrement réprimée car "perçue comme une menace au contrôle des hommes sur les femmes" (Ibid. : 246).

Dans nombre de sociétés, ce sont les capacités reproductives des femmes qui sont exploitées et entraînent les relations asymétriques entre les sexes. Paola Tabet avait démontré dans "Fertilité naturelle, reproduction forcée " (Mathieu, ed. 1985: 61-146 et Tabet 1998) que, pour obtenir les conditions optimales à l'exercice de ce contrôle de la procréation, il fallait procéder au "domptage meurtrier des femmes pour en faire des corps-outils de reproduction" (Tabet 1998: 101). C'est pourquoi, convaincue de la justesse de cette affirmation, Nicole-Claude s'intéressa aux mutilations sexuelles qui apportent la preuve que, dans les sociétés concernées, il s'agit moins de développer la sexualité des femmes que de les soumettre, que cette sexualité est cantonnée à des fins reproductrices. De sorte que dans "Relativisme culturel, excision et violences contre les femmes" (Centre d'études et de recherches inter-européennes contemporaines 1994) ${ }^{16}$, elle dénoncera le relativisme culturel pratiqué par certains de ses collègues, au motif que s'élever contre l'excision était le nouveau visage de l'impérialisme, en soulignant que, au sein même des sociétés africaines, des femmes luttaient déjà contre cette "tradition " et n'avaient pas attendu les ethnologues pour cela. 
Après le relativisme culturel, elle mit également en garde contre le postmodernisme et contre ce qu'elle a appelé «les dérives de la notion de genre» (Mathieu 2000) ${ }^{17}$, montrant que la tendance à remplacer systématiquement le mot «sexe" par le mot "genre " risquait de faire oublier qu' "à la base de l'échelle du genre, il y a bien des femelles: sexe social "femme" "(Ibid.) et que, par ailleurs, comme le dit aussi Brigitte Lhomond, "l'abandon de toute distinction entre sexe et genre entraîne le risque de naturaliser le genre" (1997). Plus fondamentalement, elle s'opposa aux théories queer auxquelles elle reprochait de "privilégier les aspects symboliques, discursifs et parodiques du genre au détriment de la réalité matérielle et historique des oppressions subies par les femmes " (Mathieu 2000, 2014 : 29-30). Dans son article «Dérive du genre/stabilité du sexe " (Mathieu 1994) ${ }^{18}$, elle en fit la démonstration, dès 1994, à partir de l'exemple des performances transgressives de la chanteuse Madonna et de sa non-influence sur les jeunes filles des milieux populaires qui se trouvent dans l'impossibilité de la prendre pour modèle dans la vie réelle.

Ce n'est que dans le courant des années 2000 que Nicole-Claude Mathieu parviendra à réaliser, partiellement, son ambition de toujours : partir des réflexions de Mauss et d'autres travaux plus récents sur la notion de personne homme et femme pour les reconsidérer à la lumière de sociétés jusqu'alors fort peu prises en compte par l'anthropologie de la parenté et de l'alliance : les sociétés matrilinéaires et/ou matri-uxorilocales (au sein desquelles le lieu de résidence d'un couple est celui de la famille de l'épouse). Elle entreprit pour cela, en collaboration avec Martine Gestin, cette somme collective qu'est Une maison sans fille est une maison morte (Mathieu, ed. 2007). Toujours avec le souci d'étudier les sujets homme et femme dans leur rapport de symétrie, son hypothèse de départ était «qu'il pourrait exister un contraste, en ce qui concerne les femmes, entre un statut de sujet quasi biologique (donc à socialiser) en sociétés patrilinéaires et, en matrilinéaires, un statut de sujet constitué d'emblée comme pleinement social-humain» (Ibid.: 2-3) ${ }^{19}$. Or, les données ethnographiques des quatorze sociétés analysées dans l'ouvrage, qui ne peuvent certes autoriser la généralisation d'autant qu'aucune d'entre elles ne se

17. Republié dans L'Anatomie politique 2 (Mathieu 2014 : 23-38).

18. Republié dans L'Anatomie politique 2 (Mathieu 2014 : 321-336).

19. L'«Introduction » à Une maison sans fille est une maison morte (Mathieu, ed. $2007: 1-53$ ) a été republiée dans L'Anatomie politique 2, sous le titre "Circulation des hommes, permanence des femmes, matriarcats imaginaires et autres curiosités...» (Mathieu 2014 : 217-271). 
rapporte aux sociétés africaines semblent confirmer qu'il ne peut en être ainsi que lorsque la résidence est effectivement matri-uxorilocale, et non lorsqu'une société matrilinéaire est à résidence virilocale. Il est même une société, celle des Kavalan de Taïwan étudiés par Pi-chen $\mathrm{Liu}^{20}$, qui présente « une image quasi inversée (par rapport aux sociétés patrilinéaires et patrilocales) de la personne femme et homme et des statuts féminin et masculin; il semble qu'on puisse parler ici d'un pouvoir collectif global des femmes" (Ibid. : 24). Les jeunes hommes kavalan entrant dans l'âge viril sont en effet assimilés à la figure d'un animal sauvage (le cerf) qu’il faut soumettre au moment où ils deviennent des hommes-maris, en les assimilant cette fois à un animal domestique (le coq) que l'épouse sacrifiera symboliquement à ses ancêtres : cette représentation kavalan de la masculinité est donc "à l'opposé de celle de nombreuses sociétés patrilinéaires " puisqu'elle contient " la symbolique du contrôle de la femme sur son mari » (Ibid.: 399). Pourtant, il ressort de la plupart des sociétés examinées - alors que l'inverse n'est pas vrai au sein des sociétés patrilinéaires et patri-virilocales -, que le pouvoir des femmes peut égaler mais jamais excéder celui des hommes qui gardent un pouvoir effectif, notamment sur les plans politique et religieux, leur permettant de posséder d'emblée un statut de sujet. De plus, même si la domination masculine y est moins forte, il apparaît en revanche que la quantité de travail qui incombe aux femmes reste toujours supérieure à celle réservée aux hommes. Cela dit, l'uxorilocalité semble préserver les femmes de certaines violences : elles ne sont jamais considérées comme impures ou dangereuses, elles ne subissent pas de mutilations sexuelles ${ }^{21}$. De leur côté, les hommes semblent également épargnés, même s'ils peuvent se plaindre du matriarcat qu'ils subissent. C'est le cas, par exemple, dans la société ngada ${ }^{22}$ où "des générations d'hommes sont passées par la transformation de fiancés travailleurs en maris paresseux juste après le mariage ", ce que Susanne Schröter décrit comme une forme de résistance passive contre le système matri-uxorilocal (Ibid. : 343)...

20. Cf. Pi-chen Liu, "Cerf-amant, coq-mari et femmes chamanes : disjonction des sexes chez les Kavalan (Taiwan)» (in Mathieu, ed. 2007 : 385-414).

21. Sauf chez les Shipibo-Conibo étudiés par Françoise Morin et Bernard Saladin d'Anglure (cf. "Excision féminine/incision masculine ou la construction de la personne chez les ShipiboConibo d'Amazonie péruvienne ", in Mathieu, ed. 2007 : 183-212), société matri-uxorilocale mais où la parenté est cognatique à forte inflexion patrilinéaire, ce qui conforte le lien qu'établit NicoleClaude Mathieu "entre l'excision et les aspects patrilinéaires des structures sociales " (Ibid.: 29). 22. Cf. Susanne Schröter, "Parenté, herméneutique et notions de genre: les facettes du pouvoir sexué chez les Ngada d'Indonésie orientale» (in Mathieu, ed. 2007 : 333-358). 
Ce tour d'horizon des travaux de Nicole-Claude Mathieu, si incomplet et réducteur soit-il, aura, je l'espère, permis de constater la cohérence, la constance et la rigueur de la pensée de cette grande anthropologue qui contribua si fortement à construire le champ des études féministes en France. Il m'a ainsi paru important de faire systématiquement figurer les titres et dates de première publication de ses articles, afin que l'on puisse juger de leur précocité. De même, en développant les prénoms de tous les auteurs cités, j'ai répondu à l'injonction de Nicole-Claude qui estimait important pour l'épistémologie des sciences sociales de savoir qui parle: un homme ou une femme? Enfin, je ne voudrais pas clore cet hommage sans mentionner la traduction que Nicole-Claude a faite du livre de Gail Pheterson, The Prostitution Prism (1996) ${ }^{23}$. De nombreuses féministes radicales ont milité, et militent toujours, contre la prostitution et, par là, contre les prostituées. Ce ne fut pas le cas de Nicole-Claude qui voyait dans la dissociation entre "femmes bien" et "femmes esclaves" ou "femmes victimes» un outil de plus dans la panoplie du sexisme. Aussi était-elle d'accord avec Gail Pheterson lorsque celle-ci écrivait :

"Les droits de l'ensemble des femmes sont indissolublement liés aux droits des prostituées parce que le stigmate de putain peut s'adresser à n’importe quelle femme pour disqualifier sa revendication à la légitimité et peut jeter la suspicion sur n’importe quelle femme accusée d'avoir pris une initiative dans le domaine économique et/ou sexuel » (2001: 151).

Cette position n'est pas restée pure écriture. Nicole-Claude s'est engagée, dès sa création en 2005, dans une association d'aide aux prostituées âgées, l'ANA ("Avec nos aînées »), à laquelle elle a consacré beaucoup de temps et de moyens matériels. Sa générosité, dont elle ne se vantait guère, était immense. Elle transparaissait aussi, nous l'avons vu, dans sa manière d'encadrer ses étudiants, car il s'agissait pour elle d'œuvrer pour la transmission de son message. Tel est le sens de son adresse aux jeunes diplômés de l'Université Laval lors de sa nomination comme Docteure en sciences sociales honoris causa:

"Même si certaines choses ont changé dans nos pays, il reste du pain sur la planche pour abolir les rapports de force qui existent entre les sexes, et je vous convie à travailler, en tant que citoyennes et citoyens, à ces transformations " (2014:11).

École des hautes études en sciences sociales Laboratoire d'anthropologie sociale, Paris mehandman@gmail.com

23. Pour la traduction de Nicole-Claude, cf. Gail Pheterson (2001). 


\section{Mathieu, Nicole-Claude}

1971 «Notes pour une définition sociologique des catégories de sexe", Épistémologie sociologique 11 : 19-39.

1989 «Identité sexuelle/sexuée/de sexe : trois modes de conceptualisation du rapport entre sexe et genre ", in Anne-Marie DauneRichard, Marie-Claude Hurtig \& Marie-France Pichevin, eds, Catégorisation de sexe et constructions scientifiques.

Aix-en-Provence, Université de Provence («Petite collection CEFUP ») : 109-147.

1991 L'Anatomie politique. Catégorisations et idéologies du sexe. Paris, Côté-femmes ("Recherches»). [Nouv. éd. : DonnemarieDontilly, Éd. iXe, 2013 ("Racine de iXe»).] 1994 "Dérive du genre/stabilité des sexes ", in Michel Dion, ed., Madonna, érotisme et pouvoir. Paris, Kimé ( $"$ Le sens

de l'histoire") : 54-70.

2000 "Sexe et genre », in Helena Irata

et al., eds, Dictionnaire critique du féminisme.

Paris, Presses universitaires de France

(«Politique d'aujourd'hui ») : 191-200.

2014 L'Anatomie politique 2. Usage,

déréliction et résilience des femmes. Paris,

La Dispute ("Le genre du monde»).

Mathieu, Nicole-Claude, ed.

1985 L'Arraisonnement des femmes.

Essais en anthropologie des sexes. Paris,

École des hautes études en sciences sociales

("Cahiers de l'Homme» 24).

2007 Une maison sans fille est une maison morte. La personne et le genre en sociétés matrilinéaires et/ou uxorilocales.

Paris, Éd. de la MsH.

\section{AUTRES RÉFÉRENCES CITÉES}

Bonte, Pierre \& Michel Izard, eds

1991 Dictionnaire de l'ethnologie et de l'anthropologie. Paris, Presses universitaires de France. [Nouv. éd. : 2000 («Quadrige»).]

Centre d'études et de recherches inter-européennes contemporaines

1994 Sexe et race. Discours et formes nouvelles d'exclusion du XIXe au XX' siècle, 9. Paris, Publ. de l'Université Paris 7-Denis Diderot : 87-102.

\section{Gestin, Martine \& Nicole-Claude Mathieu}

2010 "Claude Lévi-Strauss et (toujours) l'échange des femmes : analyses formelles, discours, réalités empiriques ", in Danièle Chabaud-Rychter et al., eds, Sous les sciences sociales, le genre. Relectures critiques, de Max Weber à Bruno Latour. Paris, La Découverte : 64-76.

\section{Godelier, Maurice}

1982 La Production des grands hommes. Pouvoir et domination masculine chez les Baruya de Nouvelle-Guinée. Paris, Fayard ("L'Espace du politique»).

\section{Lévi-Strauss, Claude}

1983 Le Regard éloigné. Paris, Plon.

\section{Lhomond, Brigitte}

1997 «D'un antinaturalisme à un a-sociologisme : comment penser les catégories de sexe et la sexualité ?", GRAAT 17 : Sexualités américaines: 33-37.

Michard-Marchal, Claire \& Claudine Ribéry 1985 «Énonciation et effets idéologiques: les objets de discours "femmes" et "hommes" en ethnologie", in Nicole-Claude Mathieu, ed., L'Arraisonnement des femmes... : 147-167. 


\section{Pheterson, Gail}

1996 The Prostitution Prism.

Amsterdam, Amsterdam University Press.

2001 Le Prisme de la prostitution. Trad. de l'anglais (États-Unis) par Nicole-Claude Mathieu. Paris, L'Harmattan ("Bibliothèque du féminisme »).

\section{Rubin, Gayle}

1975 "The Traffic in Women :

Notes on the "Political Economy" of Sex ", in Rayna Rapp Reiter, ed., Toward an Anthropology of Women. New York, Monthly Review Press : 157-210.

1998 "L'économie politique du sexe: transactions sur les femmes et systèmes de sexe/genre [trad. de l'anglais par Nicole-Claude Mathieu en collab. avec Gail Pheterson] ", Les Cahiers du CREDEF 7 [http://cedref.revues.org/171].
2010 «Le marché aux femmes : économie politique du sexe et systèmes de sexe/genre", in Surveiller et jouir. Anthropologie politique du sexe. Trad. de l'anglais (États-Unis) par Flora Bolter et al. ; éd. par Rostom Mesli. Paris, EPEL ( Les grands classiques de l'érotologie moderne ") : 23-82.

Shapiro, Harry L., ed.

1956 Man, Culture and Society.

New York, Oxford University Press.

\section{Tabet, Paola}

1979 "Les mains, les outils, les armes", L'Homme 19 (3-4) : 5-62.

1998 La Construction sociale de l'inégalité des sexes. Des outils et des corps. Paris, L'Harmattan («Bibliothèque du féminisme»).

Page de titre:

Détail d'une photographie prise en 1982, lors du dernier cours de Claude Lévi-Strauss au Collège de France

(C) Collège de France. Archives du Laboratoire d'anthropologie sociale). 